\title{
Spectroscopic diffraction phase microscopy
}

\author{
Hoa Pham, Basanta Bhaduri, Huafeng Ding, and Gabriel Popescu* \\ Quantitative Light Imaging Laboratory, Department of Electrical and Computer Engineering, \\ Beckman Institute for Advanced Science \& Technology, \\ University of Illinois at Urbana-Champaign, Urbana, Illinois 61801, USA \\ ${ }^{*}$ Corresponding author: gpopescu@illinois.edu
}

Received May 14, 2012; accepted July 13, 2012;

posted July 16, 2012 (Doc. ID 168626); published August 10, 2012

\begin{abstract}
We present spectroscopic diffraction phase microscopy (sDPM) as a method capable of measuring quantitative phase images at multiple wavelengths. sDPM uses a spatial light modulator at the Fourier plane of a lens to select desired wavelengths from the white light illumination of a grating. The quantitative phase information at different wavelengths allows us to decouple the refractive index and the thickness from the phase shift induced by biological cells. We demonstrate the capability of the setup by dispersion measurements of microsphere beads and RBCs. (c) 2012 Optical Society of America
\end{abstract}

OCIS codes: $\quad 170.1470,180.3170,170.6510,120.5050$.

Quantitative phase imaging (QPI) has been developing at an accelerated pace over the past several years [ $\underline{1}-\underline{3}]$. In QPI we measure the phase delay induced by a specimen relative to its surroundings. This phase delay is linearly related to the refractive index and thickness of the sample. For cells, this refractive index is simply proportional to concentration of proteins [4]. Recently it has been demonstrated that QPI is capable of providing nanoscale, quantitative morphological analysis as well as several clinically relevant parameters for red blood cells (RBCs) at the single cell level [5-9]. However, since the optical phase shift is a function of both thickness and refractive index, more than one measurement is needed to decouple the thickness and refractive index of the cells. One approach to solve this problem is to use two different immersion media to decouple the thickness and refractive index [10-12]. This experiment requires the cells be kept in place as the media is changed, coating of the cover slips, and a perfusion setup. Thus, such a technique may not be well suited for clinical measurements. Another approach is to measure quantitative phases at different light colors. Park et al. [13] successfully demonstrated diffraction phase microscopy (DPM) at different wavelengths using several band-pass filters. Similarly, Fu et al. [14] developed a dual-wavelength quantitative phase microscope, also based on DPM, using two independent laser sources at different wavelengths. Recently, in addition to the phase image, Mir et al. proposed to use an absorption measurement at the Soret-band wavelength [15]. Ding et al. [16] showed that quantitative phase images at three colors can be obtained simultaneously using a RGB camera.

One limitation of all of the above methods is that they either require mechanical switching of the filters [13], filter wheel [15], or manual switching of the media [17]. Furthermore, in those methods that employ the multiple wavelength approach, the number of wavelengths is limited up to three wavelengths, which limit those methods to only simple cell structures. In this Letter, we present a new method, spectroscopic diffraction phase microscopy (sDPM), that can measure quantitative phase delays induced by samples at several wavelengths. sDPM requires no moving parts in the system, and the spectroscopic measurement can be performed via full digital control. sDPM uses white light diffraction phase microscopy (wDPM) [18] and a spatial light modulator to select different wavelengths at the Fourier plane of the image. SDPM is implemented as an add-on module to a commercial microscope (Axio Observer Z1, Zeiss, in this case). The method is demonstrated by measuring the dispersion of microsphere beads and the potential for biological studies by measuring hemoglobin-induced dispersion in RBCs.

Figure 1 shows the experimental setup, where a wDPM system uses spatially coherent white light illumination, obtained from a halogen lamp.

The condenser aperture is NA $=0.09$, such that the field at the image plane is spatially coherent over the entire field of view. The illumination power at the sample plane for this NA is reduced (approximately $0.16 \mathrm{~mW}$ at the sample plane). However, this power level allows us to acquire quantitative phase images at up to 10 frames per second. An amplitude diffraction grating with a groove density of 110 grooves per millimeter placed at the image plane of the microscope generates multiple diffraction orders containing full spatial information about the image. The zero and first order beams are isolated at the Fourier plane generated by lens L1 using a mask on an amplitude spatial light modulator (SLM), as shown in Fig. 1. The SLM was obtained from an Epson Powerlite

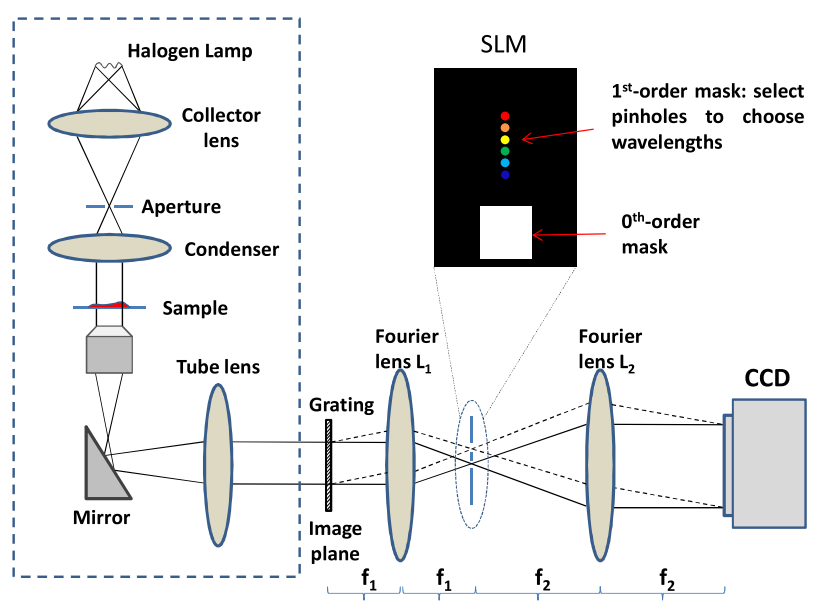

Fig. 1. (Color online) Experimental setup of sDPM. 
S5 projector, having a pixel size of $13 \mu \mathrm{m} \times 13 \mu \mathrm{m}$ and contrast ratio of $400: 1$. Figure $2 \mathrm{a}$ is an image of the Fourier plane of the setup where the SLM is placed. The key observation is that, due to the diffraction grating, the light is dispersed according to the wavelength in the first order at the Fourier plane, as shown in Fig. 2a. The dispersed signal is focused back to the same point in the image plane by the lens L2. Based on this observation, we design a series of filter masks on the SLM to select different wavelengths. In order to select the wavelengths, in the first order, a pinhole (a circular mask on the SLM) is scanned as depicted in Fig. 2b to cover the whole spectrum of the first order. Of course, the zeroth order is not dispersed in wavelengths, i.e., it maintains the white color. The lens system L1-L2 forms a highly stable MachZehnder interferometer. The filtered first-order is used as the reference beam, and the zeroth order plays the role of the imaging field. Both beams are interfered and generate a spatially modulated interference image, which is then captured by a monochrome CCD camera (Hamamatsu ORCA Flash) at the image plane. The interferogram recorded on the CCD can be used to extract the quantitative phase shifts caused by the sample via a Hilbert transform [19]. Thus by changing this SLM masks, we can measure quantitative phase information at different wavelengths. Note that the fringes observed are due to the interference between the light at the color selected by the pinhole and the white light of the zeroth order. Because of the incomplete overlap in spectra, the contrast of the fringes is reduced. However, it is more than sufficient to allow us to perform the phase reconstruction.

Throughout our experiments, we used a bright-field objective $40 \times(0.75 \mathrm{NA})$. The L1-L2 lens system, with focal lengths of $60 \mathrm{~mm}$ and $150 \mathrm{~mm}$, respectively, gives an additional magnification of $f_{2} / f_{1}=2.5$ so that the sinusoidal modulation of the image is sampled by 6 CCD pixels per period. For this objective, the diameter of the pinhole (first order mask) at the Fourier plane is $200 \mu \mathrm{m}$.

Figure 2c shows the spectrum of the first order diffraction (the pink outer envelope) and the spectra
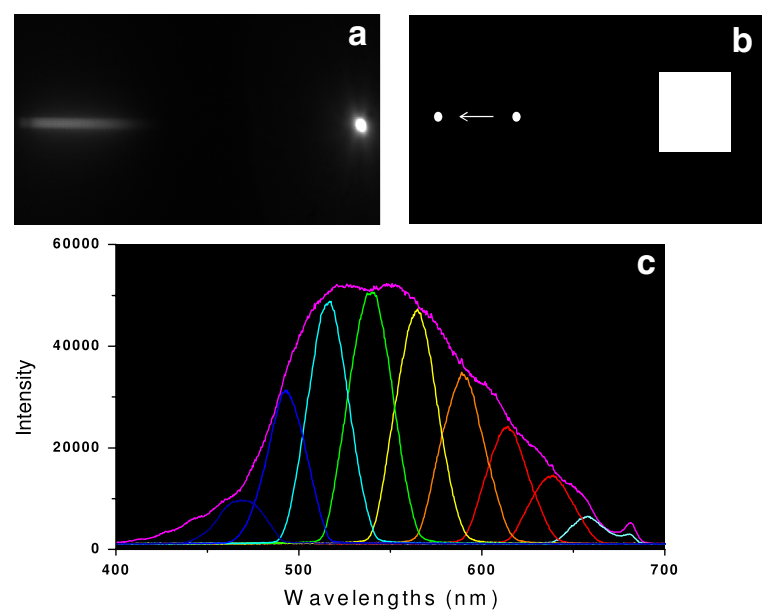

Fig. 2. (Color online) a, image captured at the Fourier plane. b, scanning filters on the SLM. c, first order diffraction spectrum (outer envelope) and spectra at different wavelengths. obtained from different positions of the pinhole measured by Ocean Optics USB2000+ Fiber Optic Spectrometer. The FWHM of each spectrum is $28 \mathrm{~nm} \pm 1 \mathrm{~nm}$. The mean wavelength of each spectrum is then used to calculate the expected phase shift corresponding to each pinhole position.

In order to calibrate the system, we first measured polystyrene microsphere beads immersed in immersion oil (Zeiss). The phase shift measured by the system is related to the refractive index and thickness of the sample as

$$
\Delta \phi(x, y ; \lambda)=k_{0} \Delta n(\lambda) h(x, y)
$$

where $k_{0}=2 \pi / \lambda$ and $\lambda$ is the mean wavelength, $h$ is the thickness, and $\Delta n$ is the difference between the refractive index of the sample and that of the surrounding media. When calculating the expected phase shifts, we also took into account the dispersion of the immersion oil. Figures $3 \mathrm{a}-3 \mathrm{~d}$ show the reconstructed phase maps of the $2.9 \pm \overline{0.1} \overline{4} \mathrm{~m}$ polystyrene bead at four different wavelengths $(565,589,613$, and $640 \mathrm{~nm})$. Figure 3e shows the average phase values (red dashed curve) for 10 polystyrene microspheres with the error bar indicating standard deviation error. The expected phase shift is shown by the blue solid curve. The measured dispersion agrees very well with the expected dispersion [17].

Next we demonstrate an important application of sDPM, by measuring the dispersion of RBCs. Before imaging, the whole blood was diluted with Coulter LH series diluent (Beckman-Coulter). A sample chamber is created by punching a hole in double sided scotch tape and sticking one side of the tape onto a cover slip. The sample is then pipetted into the chamber created by the hole and it is sealed on the top using another cover slip [19]. For RBCs, the phase shift in Eq. (1) can be rewritten as

$$
\Delta \phi(x, y ; \lambda)=k_{0}\left[\beta(\lambda) C(x, y)+\Delta n_{w s}(\lambda)\right] h(x, y),
$$
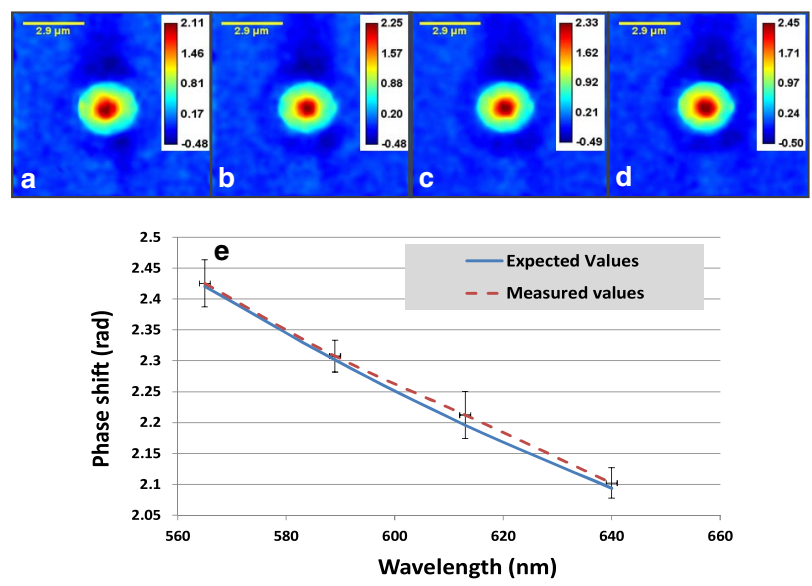

Fig. 3. (Color online) Dispersion relation of $3 \mu \mathrm{m}$ beads in Zeiss immersion oil. a-d, reconstructed phase maps at different wavelengths. e, measured (dashed curve) and expected (solid curve) dispersion curves. The error bars indicate standard deviation $(N=10)$. 

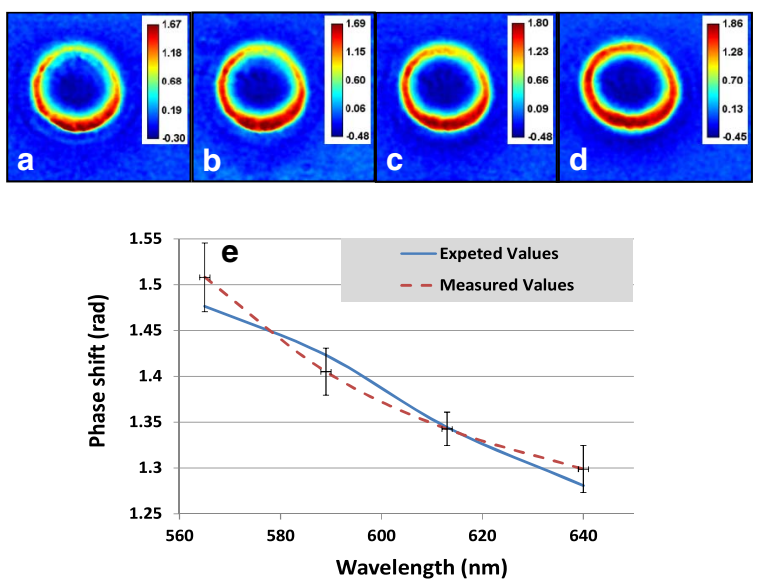

Fig. 4. (Color online) Dispersion of RBCs. a-d, reconstructed phase maps in radians at different wavelengths. e, dispersion relation. Expected phase shift of $2 \mu \mathrm{m}$ height RBC (solid curve) and average of normalized phase shifts of measured RBC (dashed curve). The error bar indicates the standard deviation $(N=10)$.

where $\beta$ is the refractive increment of protein in $\mathrm{mL} / \mathrm{g}$, i.e., the rate of change of the refractive index versus the concentration of the solution, $C$ is the concentration in $\mathrm{g} / \mathrm{mL}$, and $\Delta n_{w s}$ is the difference between the refractive index of water and the surrounding media. Since we can measure the phase shifts at multiple wavelengths, we can solve for $C$ and $h$ simultaneously. In order to compute the expected phase shifts, we use $C=32 \mathrm{~g} / \mathrm{dL}$ and data for the refractive increment $\beta$ from the literature [20]. Figures $4 \mathrm{a}-\underline{4 d}$ present phase maps of an RBC at the same four different wavelengths. The phase maps were then thresholded to segment RBCs and the phase shift for each cell was averaged over the cell. Figure 4e shows the dispersion curve associated with these measurements. The expected phase shift values (solid curve) were calculated for a $2 \mu \mathrm{m}$ thick RBC; different thickness only shifts this curve vertically. In order to remove the errors due to variations in RBC thickness values, we normalized the phase values of each cell to the mean of the end points of the measured dispersion. Then the phase value at each wavelength was averaged over $10 \mathrm{RBC}$ cells as shown in the red dash line in Fig. 4e. The error bar indicates the standard deviation error. The results show that the measured phase shifts agree very well with the expected dispersion.

In summary, we developed sDPM, a method capable of measuring quantitative phase maps at multiple wavelengths. By adding simple optics, we can convert a commercial microscope into a quantitative phase spectroscopy instrument. To measure phase at different wavelengths, we simply scan the pinhole mask on the
SLM at the Fourier plane. This method has the advantage of using existing microscopes with minimal changes, instead of using either band-pass filters [13] or two different laser sources [14]. Measurements on polystyrene microspheres show that the results agree well with theory. We also demonstrate the measurement of the dispersion of single RBCs. This technique will be very useful for studying RBCs as well as characterizing various blood disorders.

This research was supported in part by the National Science Foundation (grants CBET 08-46660 CAREER, CBET-1040462 MRI) and the National Cancer Institute (grant R21 CA147967-01).

\section{References}

1. G. Popescu, Quantitative Phase Imaging of Cells and Tissues (McGraw-Hill, 2011).

2. E. Cuche, F. Bevilacqua, and C. Depeursinge, Opt. Lett. 24, 291 (1999).

3. A. Barty, K. A. Nugent, D. Paganin, and A. Roberts, Opt. Lett. 23, 817 (1998).

4. R. Barer, J. Opt. Soc. Am. 47, 545 (1957).

5. Y. K. Park, C. A. Best, T. Auth, N. S. Gov, S. A. Safran, G. Popescu, S. Suresh, and M. S. Feld, Proc. Natl. Acad. Sci. USA 107, 1289 (2010).

6. Y. Park, C. A. Best, K. Badizadegan, R. R. Dasari, M. S. Feld, T. Kuriabova, M. L. Henle, A. J. Levine, and G. Popescu, Proc. Natl. Acad. Sci. USA 107, 6731 (2010).

7. G. Popescu, Y. Park, R. R. Dasari, K. Badizadegan, and M. S. Feld, Phys. Rev. E 76 (2007).

8. G. Popescu, Y. Park, N. Lue, C. Best-Popescu, L. Deflores, R. R. Dasari, M. S. Feld, and K. Badizadegan, Am. J. Physiol. Cell Physiol. 295, C538 (2008).

9. N. T. Shaked, L. L. Satterwhite, M. J. Telen, G. A. Truskey, and A. Wax, J. Biomed. Opt. 16, 895 (2011).

10. B. Rappaz, A. Barbul, Y. Emery, R. Korenstein, C. Depeursinge, P. J. Magistretti, and P. Marquet, Cytometry Part A 73A, 895 (2008).

11. B. Rappaz, F. Charriere, C. Depeursinge, P. J. Magistretti, and P. Marquet, Opt. Lett. 33, 744 (2008).

12. B. Rappaz, P. Marquet, E. Cuche, Y. Emery, C. Depeursinge, and P. J. Magistretti, Opt. Express 13, 9361 (2005).

13. Y. Park, T. Yamauchi, W. Choi, R. Dasari, and M. S. Feld, Opt. Lett. 34, 3668 (2009).

14. D. Fu, W. Choi, Y. J. Sung, Z. Yaqoob, R. R. Dasari, and M. Feld, Biomed. Opt. Express 1, 347 (2010).

15. M. Mir, K. Tangella, and G. Popescu, Biomed. Opt. Express 2, 3259 (2011).

16. H. F. Ding and G. Popescu, Opt. Express 18, 1569 (2010).

17. http://refractiveindex.info/.

18. B. Bhaduri, H. Pham, M. Mir, and G. Popescu, Opt. Lett. 37, 1094 (2012).

19. T. Ikeda, G. Popescu, R. R. Dasari, and M. S. Feld, Opt. Lett. 30, 1165 (2005).

20. M. Friebel and M. Meinke, Appl. Opt. 45, 2838 (2006). 